\title{
Measurement of apparent depth in a Lissajous pattern
}

WALTER H. RIEGE

Twenty-four Ss participated in a haploscopic matching procedure to measure the distance of apparent near point and far point in a rotating Lissajous figure which was seen on the oscilloscope screen. Among $S$ s the variability was high in their estimates of apparent depth. Herein, the effect of learning was a more efficient aid in the judgmental task than the $90^{\circ}$ change in plane of rotation of the figure.

The classical cues of depth perception are described to derive predominantly from the stationary display of objects. A rigid geometrical transformation of a visual pattern, on the other hand, may yield information concerning the depth of the figure represented by that pattern which is not available in its stationary view (Braunstein, 1962). The sharply defined shadow of a stationary object, projected onto a plane, may assume a variety of two-dimensional appearances, but the transforming shadow of an object moved or rotated in one of its three planes induces a more striking impression: The shadow acquires a substantial appearance and looks itself an object moving in space (Metzger, 1934).

An intriguing demonstration of depth in moving patterns is the visual presentation of so called Lissajous figures. The 19th century French scientist, Jules A. Lissajous (1822-1880), observed that closed curves result from the combination of two simple harmonic motions. He arranged two tuning forks at right angles with mirrors pasted to their tips which reflected light onto a screen. With equal frequencies, the reflected light beams would produce a circle, seen alternately rotating around each of two perpendicular diagonal axes.

Lissajous patterns result also on the screen of an oscilloscope when the outputs of two oscillators are connected, one to the vertical, the other to the horizontal input. Frequencies adjusted in simple numerical ratio will give a stationary pattern display. By slight mistuning of the frequencies, the pattern can be set into apparent rotation. Rotation about a horizontal (HA) or about a vertical (VA) axis depends upon which input receives the higher frequency. Stationary patterns are seen two dimensionally, but Lissajous patterns in motion seem to lack reference points, and resemble three dimensional rotating wire figures.

Previous studies seem to have been concerned with the depth effect per se. If one must assume, however, that not the pattern but the continuous transformation is the stimulus in its own right (Gibson, 1966), then constant transformation, i.e., HA or VA rotation, should elicit the same depth effect. It had been postulated that HA rotation preempted the depth effect over VA rotation (Braunstein, 1966), so that no invariant distance judgments could be hypothesized for the present experiment.

Method

Two audio oscillators were connected to horizontal and vertical inputs of an oscilloscope (CRT) and produced a three-looped Lissajous pattern on the screen. To avoid interference from the ac line frequency, only the frequencies 60 and $90 \mathrm{~Hz}$ were used. By exchanging these two frequencles between the oscillators and by increasing the higher frequency slightly (by $0.5 \mathrm{~Hz}$ ), either a VA or a HA rotation of the figure was obtained. In this way a green phosphorescent Lissajous figure of screen size $5 \times 5 \mathrm{~cm}$ was seen by Ss from a distance of $75 \mathrm{~cm}$, measured from the CRT to a Styrofoam chinrest.

Twenty-four male and female students from the introductory psychology class participated to measure individually the apparent distance by haploscopic matching. The reference scale for the depth judgments consisted basically of a movable, red-dot light source, a pair of convex lenses, a single-prism mirror, and two crossed polarizing fliters, all mounted on an optic bench with metric scaling. The optic bench was fastened to a table $55 \mathrm{~cm}$ in front of the chinrest so that the red-dot light moved perpendicularly to S's line of sight, and was projected through the filters and mirror to the S's left eye view only, while $S$ could see the Lissajous figure with the right eye only.

Asked for the description of the right eye view, after $10 \mathrm{~min}$ dark adaptation, all Ss reported seeing a three-looped, three-dimensional rotating figure. From such descriptions $E$ explained that there must necessarily exist in the figure a near point and a far point with reference to the axis congruent with the line of sight. Simultaneously observing both the moving red dot approaching or receding and the near point of the flgure, each $S$ estimated where both coincided in distance along the line of sight. A similar estimate was obtained for the far point. Depth judgments were given in 45 trials under the method of constant stimulus differences. The same procedure was repeated with the figure shifted $90^{\circ}$, i.e., when the rotation was changed from $\mathrm{HA}$ to VA or vice versa. The Ss were counterbalanced for order of presenting the HA or the VA rotation first. In addition, for each of the 
two estimates of the two points, four series under the method of limits were given, and the boundaries of the uncertainty interval recorded.

Results

Using the normal-interpolation process, averaged distance values for near and for far point in the Lissajous figure were obtained from the constant-stimuli trials. Equal-distance responses under the method of limits were computed as the geometric mean of the uncertainty interval, since discrimination was rather poor and the distance between the two limen could not be assumed to be two DL.

The differences between mean far and near point values as indicators of apparent depth were highly variable. Only five Ss showed consistent distance judgments differences for the change from $\mathrm{HA}$ to VA rotation, if a $10 \%$ variation was accepted. In order to overcome the extensive Ss' deviation and to evaluate judged depth, the metric data were transformed into the ratio of far to near point. The depth ratios immediately point out the effect of order of presentation on judged depth. The rotation condition presented second, in both groups, resulted in larger estimates of far and near point intervals. The Group HA-VA showed larger ratios and larger differences for the VA rotation of the pattern than for the HA rotation, a fact that was exactly reversed for all but three Ss of the Group VA-HA. An analysis of variance found an interaction between groups and rotation condition to be significant at the 0.01 level $(F=9.59, \mathrm{df}=1 / 22)$. No difference was demonstrated to exist in the depth ratios taken from the repeated measures of each $S$ under the HA or under the VA rotation presentation. On the other hand, both measures were found in both groups to be only slightly correlated with each other (Group HA-VA, $r=+0.387$; Group VA-HA, $r=+0.233$ ), so that an equality of depth effects could not be inferred. The variability of Ss of Group HA-VA was slightly larger $(S D=0.176)$ than that for the VA-HA Ss $(\mathrm{SD}=0.114)$.

\section{Discussion}

Two-dimensional Lissajous patterns, generated from mistuned sinusoldal voltage changes on an oscilloscope screen, were seen as the rotation of rigid three- dimensional figures. With haploscopic matching, human observers were rarely able to reliably quantify the apparent depth in the figure. The difficulty seemed to lie in the task for the Ss rather than in the measuring technique. The distance matching of the two monocular images were determined by different perceptual cues. Variable retinal angle of the moving red dot at the left eye view had to be compared with an inexplicit and stationary near or far point in the Lissajous figure. Depth was induced by the constant rotation of the pattern, since every possible visual cue to a surface as the carrier of the pattern of light brought to the right eye had been excluded. To most $\mathrm{Ss}$, the figure appeared two-dimensional as soon as rotation stopped and the lines of the pattern remained congruent for a brief period.

Since Helmholtz there has been agreement that movement parallax is a forceful secondary cue for perceiving the third dimension, but in monocular vision it may provide a basis for ambiguities in depth judgments. In the present study, the apparent rotation offered little aid in the distance judgments. The problem of veridicality was not pertinent, because Ss dealt with object depth where no object was specifiable.

Learning to judge distances of the hypothetical points improved with trials. The order of figure rotation, contrary to Braunstein's (1966) conclusion, was more important than axis of rotation; the apparent depth was consistently perceived to be larger in the second condition. Furthermore, $16 \mathrm{Ss}$ reported to be aided by a gradual fusion of the haploscopic images, which could not be ascribed to convergence but to integration at a more central neural stage. Perceived threedimensionality appears subservient to $\mathrm{Ss}^{\prime}$ criteria, and to the extent that no other visual cues than pattern transformations are avallable, depth is ambiguous.

\section{References}

Braunstein, M. L. The perception of depth through motion. Psychol. Bull., 1962, 59, 422-433.

Braunstein, M. L. Sensitivity of the observer to transformations of the visual field. J. exp. Psychol., 1966, 72, 683-689.

Gibson, J. J. The senses considered as perceptual system. Boston: Houghton Mifflin Co., 1966.

Metzger, $W$. Tiefenerscheinungen in optischen Bewegungsfeldern (1934). Psychologische Forschung, 1935, 20, 195-260. 